\title{
Efeitos do Estado de Humor sobre os Comportamentos Alimentares Inadequados de Atletas de Atletismo
}

\author{
Leonardo de Sousa Fortes \\ Universidade Federal de Pernambuco ${ }^{1}$ \\ Juliana Fernandes Filgueiras \\ Clara Mockdece Neves \\ Universidade Federal de Juiz de Fora \\ Sebastião Sousa Almeida \\ Universidade de São Paulo \\ Maria Elisa Caputo Ferreira \\ Universidade Federal de Juiz de Fora
}

\begin{abstract}
RESUMO - O objetivo do presente estudo foi analisar os efeitos do estado de humor sobre os comportamentos alimentares inadequados (CAI) em atletas. Participaram 68 atletas de atletismo, do sexo feminino, com idades entre 12 e 17 anos. Utilizaramse o Eating Attitudes Test (EAT-26) e a Escala de Humor de Brunel (BRUMS) para avaliar os CAI e o distúrbio total do humor (DTH), respectivamente. Dois modelos de regressão linear não indicaram influência estatisticamente significativa da BRUMS nas subescalas Dieta $(p=0,42)$ e Bulimia e Preocupação com Alimentos $(p=0,81)$ do EAT-26. Ao contrário, a BRUMS ( $p=0,04)$ demonstrou impacto significante (7\%) sobre os escores da subescala Autocontrole Oral. Concluiu-se que as atletas com DTH elevado podem estar mais susceptíveis à influência ambiental para a ingestão alimentar.
\end{abstract}

Palavras-chave: alteração do humor, transtornos alimentares, atletas do sexo feminino, atletismo

\section{Effects of Mood State on Inappropriate Eating Behavior of Track and Field Athletes}

\begin{abstract}
The goal of the present study was to analyze the effects of mood state on inappropriate eating behavior (IEB) of athletes. The participants were 68 female track and field athletes, with ages between 12 and 17 years. The Eating Attitudes Test (EAT-26) and the Brunel Mood Scale (BRUMS) were used to evaluate the IEB and total mood disturbance (TMD), respectively. Two linear regression models indicated no statistically significant influence of BRUMS on Diet $(p=0,42)$ and on the Bulimia and Food Preoccupation $(p=0,81)$ subscales of the EAT-26. Rather, BRUMS $(p=0,04)$ showed a significant impact (7\%) on Oral Selfconttrol subscale scores. It was concluded that athletes with high TMD might be more susceptible to environmental influence upon food intake.
\end{abstract}

Keywords: change in mood, eating disorders, female athletes, track and field

Os comportamentos alimentares inadequados (CAI) são considerados um dos principais fatores de risco para o desencadeamento dos transtornos alimentares (TA) (De Bruin, Oudejans, \& Bakker, 2007; Scoffier, Woodman, \& D’Arripe-Longueville, 2011). A restrição e a compulsão alimentar, o uso de laxantes e diuréticos, a autoindução de vômitos e o uso de esteroides anabólicos são alguns exemplos desses comportamentos (Rosendahl, Bormann, Aschenbrenner, Aschenbrenner, \& Strauss, 2009). De acordo com a literatura científica, a prevalência dos CAI no público adolescente é considerada alta, variando de 20 a 35\% (Coelho, Soares, \& Ribeiro, 2010; Fortes \& Ferreira, 2011). Alguns autores ressaltam que essa prevalência é ainda maior no sexo feminino quando comparado ao masculino (Durme, Goossens \& Braet, 2012; Haase, 2011). Ainda, evidências

1 Endereço para correspondência: Universidade Federal de Pernambuco, Centro Acadêmico de Vitória, Núcleo de Educação Física e Ciências do Esporte, Rua Alto do Reservatório, Bela Vista, Vitória de Santo Antão, PE, Brasil, CEP: 55608-680,E-mail: leodesousafortes@hotmail.com indicam que as atletas emitem com maior frequência esses comportamentos em relação às não atletas (De Bruin et al., 2007; Rosendahl et al., 2009).

Salienta-se que o âmbito esportivo competitivo é repleto de cobranças e exigências no que tange a otimização do desempenho atlético (Fortes, Almeida, Laus \& Ferreira, 2012; Reiking \& Alexander, 2005; Rosendahl et al., 2009). É comum os treinadores, os patrocinadores e até mesmo os pais cobrarem excessivamente bons resultados esportivos das atletas. No entanto, ressalta-se que as exigências variam em função do tipo de modalidade esportiva. Por exemplo, é considerado fundamental que as atletas de esportes de orientação estética (e.g., ginástica artística, nado sincronizado e saltos ornamentais) tenham morfologia magra e esbelta (De Bruin et al., 2007; Fortes, Neves, Filgueiras, Almeida, \& Ferreira 2013). Por outro lado, nos esportes de potência (e.g., natação e atletismo) é exigido um corpo forte com musculatura delineada (Fortes \& Ferreira, 2011; Gomes, Martins, \& Silva, 2011). Ao contrário, para os esportes com divisão por classe de peso (e.g. judô, tae-kwon- 


\section{Método}

do e boxe), exige-se controle do peso corporal (Filaire, Rouveix, Pannafieux, \& Ferrand., 2007). Evidências têm demonstrado que as atletas de esportes com características predominantemente estéticas são mais susceptíveis aos CAI quando comparadas às atletas de outras modalidades esportivas (De Bruin et al., 2007; Fortes \& Ferreira, 2011). Todavia, pouca atenção tem sido direcionada para as atletas de esportes de potência.

Segundo Fortes, Almeida e Ferreira (2012), existem diversos fatores associados aos CAI em atletas, tais como: insatisfação corporal, baixa autoestima, idade, percentual de gordura elevado, processo maturacional, traços da personalidade, entre outros. Contudo, alguns pesquisadores recomendam controlar a insatisfação corporal, a idade e o percentual de gordura nas análises dos dados em investigações que busquem analisar os CAI em razão de outras variáveis (Fortes \& Ferreira, 2011; Fortes, Oliveira \& Ferreira, 2012). Esse procedimento visa isolar os efeitos da insatisfação corporal, da idade e do percentual de gordura sobre os CAI, tornando os achados mais confiáveis.

Costarelli e Stamou (2009) e Gomes et al. (2011) sugerem outros fatores que podem influenciar a adoção de CAI entre as atletas. Um deles é o estado de humor, definido como um estado emocional de duração variável que transita entre dois polos extremos, um eufórico e outro apático, modificando de acordo com as circunstâncias ambientais (Vieira et al., 2010). A busca nas principais bases de dados de artigos científicos (e.g., Web of Science, Scopus, Medline e SciELO) não indicou a existência de pesquisas que tenham analisado a relação entre o estado de humor e os CAI em atletas.

Alguns achados demonstram que o estado de humor pode ser negativamente alterado em razão da intensidade do treinamento físico, da participação em competições ou da periodização do treinamento (Vieira et al., 2010; Werneck \& Navarro, 2011). Sendo assim, emoções como raiva, fadiga e depressão podem influenciar a adoção dos CAI em atletas do sexo feminino. Assim, caso se evidencie relação entre CAI e emoções negativas, os treinadores poderão se atentar para as mudanças de comportamentos alimentares das suas atletas após sessões intensas de treinamento, competições ou períodos pré-competitivos. Nesse sentido, a detecção precoce de alterações no comportamento alimentar em jovens atletas poderia prevenir o surgimento de TA.

Ademais, constata-se uma escassez de investigações no Brasil concernentes aos fatores relacionados com os CAI no âmbito esportivo competitivo. De fato, pouco é conhecido a respeito da gênese dos CAI em atletas brasileiros. Diante desse quadro, o objetivo do presente estudo foi analisar os efeitos do estado de humor sobre os CAI em jovens atletas. Considerando que 1) a maioria dos estudos de CAI utiliza amostras com atletas de modalidades esportivas diversificadas, o que dificulta gerar conclusões sobre possíveis relações entre CAI e esportes específicos, 2) pouco se sabe sobre a incidência de CAI em esportes de potência, e 3) a prevalência de CAI é maior em atletas do sexo feminino, o presente estudo investigou os efeitos do estado de humor sobre os CAI em atletas do sexo feminino de uma única modalidade esportiva, a saber, atletismo.
Esta pesquisa foi submetida ao Comitê de Ética e Pesquisa em Seres Humanos da Faculdade de Filosofia, Ciências e Letras da Universidade de São Paulo (USP) e foi desenvolvida somente após a sua aprovação (CAE - 05166712.8.0000.5407). Os responsáveis, assim como as atletas, assinaram um Termo de Consentimento Livre e Esclarecido (TCLE), que explicava os objetivos e procedimentos do estudo. Foi garantido o anonimato às participantes e o total sigilo no tratamento dos dados.

\section{Participantes}

Trata-se de um estudo transversal, cuja amostra, selecionada por conveniência, constituiu-se de atletas do sexo feminino de algumas modalidades do atletismo ( $100 \mathrm{~m}$ rasos, $200 \mathrm{~m}$ rasos, $100 \mathrm{~m}$ com barreiras, $400 \mathrm{~m}$ com barreiras, salto com vara, salto em distância, salto em altura e lançamento de dardo). As atletas eram pertencentes a clubes da cidade de São Paulo, SP, com idades entre 12 e 17 anos.

Para serem incluídas na pesquisa, as atletas deveriam apresentar o TCLE assinado pelo responsável; treinar por, no mínimo, cinco dias na semana e com duração mínima de 2 $\mathrm{h} /$ treino na sua respectiva modalidade; ter participado de, ao menos, uma competição de dimensão regional durante o ano de 2013; e aceitar participar voluntariamente, respondendo os questionários e participando das avaliações antropométricas.

Desse modo, a amostra da presente investigação foi composta por 79 atletas adolescentes do sexo feminino. No entanto, 11 meninas foram excluídas do estudo por não responderem os questionários em sua totalidade. Por fim, a pesquisa contou com 68 esportistas (10 dos $100 \mathrm{~m}$ rasos, sete dos $200 \mathrm{~m}$ rasos, oito dos $100 \mathrm{~m}$ com barreiras, oito dos $400 \mathrm{~m}$ com barreiras, 10 do salto com vara, nove do salto em distância, oito do salto em altura e oito do lançamento de dardo), com média de 15,23 $( \pm 1,74)$ anos para idade e 18,39 $( \pm 2,43)$ para o percentual de gordura.

\section{Instrumentos}

Para avaliar os CAI foi aplicado o Eating Attitudes Test (EAT-26), em sua versão validada para o sexo feminino por Bighetti, Santos, Santos e Ribeiro (2004), apresentando consistência interna igual a 0,82. Para o presente estudo, o instrumento apontou boa consistência interna, representada por $\alpha=0,84$. O questionário é composto por 26 questões distribuídas em três fatores: 1) dieta - diz respeito à recusa patológica a alimentos com alto teor calórico e preocupação com a aparência física; 2) bulimia e preocupação com os alimentos - refere-se a episódios de compulsão alimentar, seguidos por comportamentos purgativos para perda/controle de peso corporal; e 3) autocontrole oral - reflete o autocontrole em relação à comida e avalia as forças ambientais e sociais estimulantes à ingestão alimentar. $\mathrm{O}$ escore final do EAT26 é dado pela soma de seus itens e o ponto de corte para comportamento alimentar de risco para TA é 21, ou seja: pontuação menor que 21 indica comportamento desfavorável 
e pontuação maior ou igual a 21 representa atitudes favoráveis ao desenvolvimento de TA.

O estado de humor foi avaliado pela Escala de Humor de Brunel (BRUMS), em sua versão validada para atletas adolescentes brasileiros (Rohlfs et al., 2008). Embora Rohlfs et al. (2008) não tenham avaliado a consistência interna da BRUMS, para a presente amostra foi encontrado valor de alpha de Cronbach de 0,83, representativo de boa consistência interna. A BRUMS contém 24 indicadores simples de humor, distribuídos em seis subescalas: tensão $(T)$, depressão $(\mathrm{D})$, raiva $(\mathrm{R})$, vigor $(\mathrm{V})$, fadiga $(\mathrm{F})$ e confusão mental (CM). Cada subescala contém quatro itens em escala do tipo Likert $(\mathrm{Nada}=0$; Um pouco $=1$; Moderadamente $=2$; Bastante $=3$; Extremamente $=4$ ) e o escore pode variar de 0 a 16. As subescalas T, D, R, F e CM são consideradas os fatores negativos do humor, sendo o $\mathrm{V}$ considerado o fator positivo. O distúrbio total de humor (DTH) é calculado pela soma dos fatores negativos, subtraindo o escore do fator positivo. Ao resultado final do DTH foi somado 100 para que não houvessem resultados negativos. Utilizou-se a mediana (101) da BRUMS para classificar atletas com alto $(\geq 101)$ e baixo DTH $(<101)$.

Para avaliar a insatisfação corporal foi aplicado o Body Shape Questionnaire (BSQ), em sua versão validada para a população de adolescentes brasileiros (Conti, Cordás, \& Latorre, 2009). O instrumento apresenta boa consistência interna (alpha de Cronbach $[\alpha]=0,96)$ e coeficiente de correlação entre os escores do teste-reteste significativo $(0,89$ para meninas). Para amostra do presente estudo, identificouse valor de $\alpha=0,94$, demonstrando boa consistência do instrumento. O questionário autoavaliativo é composto por 34 perguntas em escala tipo Likert, relacionadas à preocupação que a jovem apresenta com o seu peso e com a sua aparência física. A avaliada aponta com que frequência, nas últimas quatro semanas, vivenciou os eventos propostos pelas alternativas e o escore final é dado pela soma total dos itens, sendo que quanto maior o escore, maior a insatisfação com o corpo. A classificação dos resultados do BSQ para adolescentes é dividida em quatro níveis de insatisfação corporal, sendo: pontuação abaixo de 80 indica ausência de insatisfação; entre 80 e 110, insatisfação leve; entre 110 e 140, insatisfação moderada; e pontuação igual ou acima de 140 indica grave insatisfação corporal. Em razão de algumas evidências apontarem a influência da insatisfação corporal sobre o comportamento alimentar de risco para TA (Fortes \& Ferreira, 2011; Fortes, Oliveira et al., 2012), os escores do BSQ foram inseridos como covariável em algumas análises estatísticas da presente investigação.

Para o cálculo do percentual de gordura foi utilizado o protocolo para adolescentes desenvolvido por Slaughter et al. (1988). Foram aferidas as dobras cutâneas triciptal e subescapular, de acordo com as padronizações determinadas pela Internacional Society for Advancement for Kineanthropometry (2012), a partir de um compasso (adipômetro) científico da marca LANGE®) (Cambridge Scientific Industries Inc.), com precisão de $1 \mathrm{~mm}$. As medidas foram tomadas de forma rotacional e coletadas três vezes, sendo considerada a média dos valores. Em virtude dos achados de algumas investigações indicarem influência da gordura corporal sobre o comportamento alimentar de risco para TA (De Bruin et al., 2007; Fortes \& Ferreira, 2011), o percentual de gordura foi inserido como covariável em algumas análises estatísticas no presente estudo.

\section{Procedimento}

A priori, os pesquisadores responsáveis entraram em contato com os treinadores de atletismo de diversos clubes da cidade de São Paulo/SP. Os procedimentos, bem como os objetivos do estudo, foram devidamente explicados e foi solicitada autorização para que a equipe participasse da pesquisa.

Após o consentimento dos treinadores, foi realizada uma reunião com cada equipe para que as atletas fossem esclarecidas sobre todos os procedimentos éticos da investigação. Nesse encontro, também foi entregue o TCLE para que os pais ou responsáveis autorizassem por escrito (através da assinatura do termo) a participação de suas filhas.

A coleta dos dados foi realizada em dois momentos distintos, sempre pelo mesmo pesquisador, e em salas adequadas disponibilizadas pelos clubes participantes. No primeiro encontro, as atletas responderam aos questionários (EAT-26, BRUMS e BSQ), e no segundo encontro foi realizada a avaliação das medidas antropométricas (dobras cutâneas). Assim, as meninas receberam a mesma orientação verbal e eventuais dúvidas foram esclarecidas. Também constavam nos questionários orientações escritas sobre o preenchimento dos mesmos. Durante a aplicação não houve comunicação entre as atletas e não se delimitou tempo para o preenchimento dos questionários.

\section{Análise estatística}

O teste Shapiro Wilk foi conduzido e demonstrou que a distribuição normal dos dados não foi violada. Sendo assim, utilizaram-se medidas de tendência central (média) e dispersão (mínimo, máximo e desvio padrão) para descrever as variáveis da pesquisa (EAT-26, BRUMS, BSQ, idade e percentual de gordura). Utilizou-se a mediana (101) da BRUMS para classificar atletas com alto $(\geq 101)$ e baixo $(<101)$ DTH. Conduziram-se três modelos da regressão linear múltipla stepwise: (1) para averiguar a influência da BRUMS, do BSQ e do percentual de gordura nos escores da subescala Dieta do EAT-26; (2) para identificar a influência da BRUMS, do BSQ e do percentual de gordura nos escores da subescala Bulimia e Preocupação com Alimentos do EAT-26; e (3) para analisar a influência da BRUMS, do BSQ e do percentual de gordura nos escores da subescala Autocontrole Oral do EAT-26. Por fim, a análise multivariada de covariância (MANCOVA), utilizando a idade, o BSQ e o percentual de gordura como covariáveis, foi aplicada para comparar os escores das subescalas do EAT-26 em função dos grupos estabelecidos a partir da mediana da BRUMS. O teste post hoc de Bonferroni foi utilizado para identificar a localização das diferenças estatísticas. Todos os dados foram tratados no software SPSS 17.0, adotando-se nível de significância de 5\%. 


\section{Resultados}

Os achados referentes à aplicação do EAT-26 evidenciaram que aproximadamente $14 \%(\mathrm{n}=10)$ das atletas demonstraram comportamento alimentar de risco para TA. Em adição, os resultados apontaram que cerca de $30 \%(n=20)$ das adolescentes apresentaram algum nível de insatisfação corporal avaliado pelo BSQ: $25 \%(\mathrm{n}=17)$ mostraramse levemente insatisfeitas e $5 \%(n=3)$, moderadamente insatisfeitas com o corpo. Além disso, quase $47 \%(\mathrm{n}=21)$ da amostra indicou alto DTH (BRUMS $\geq 101$ ).

Tabela 1. Valores descritivos (mínimo, máximo, média e desvio padrão) das variáveis do estudo.

\begin{tabular}{ccccc}
\hline Variável & Mínimo & Máximo & Média & DP \\
\hline EAT-26 & 0 & 29 & 10,51 & 7,96 \\
BRUMS & 91 & 136 & 104,33 & 9,28 \\
BSQ & 38 & 119 & 67,45 & 18,76 \\
Idade (anos) & 12 & 17 & 15,23 & 1,74 \\
$\% \mathrm{G}$ & 13 & 23 & 18,04 & 2,88 \\
\hline
\end{tabular}

$\mathrm{DP}=$ Desvio Padrão; EAT-26 $=$ Eating Attitudes Test; BRUMS $=$ Escala de Humor de Brunel; BSQ = Body Shape Questionnaire; $\% \mathrm{G}=$ Percentual de Gordura.

O modelo de regressão apresentado na Tabela 2 demonstrou influência somente do BSQ $\left(F_{(1,67)}=23.04\right.$; $p=0,001)$ e do percentual de gordura $\left(F_{(1,67)}=7,66 ; p=0,03\right)$ sobre os escores da subescala Dieta do EAT-26. Ao contrário, a $\operatorname{BRUMS}\left(F_{(1,67)}=0,7 ; p=0,37\right)$ não indicou influência estatisticamente significativa. Esses resultados indicam que a insatisfação corporal e o percentual de gordura explicaram, respectivamente, $63 \%$ e $30 \%$ da variância da restrição de ingestão de alimentos altamente calóricos por atletas do sexo feminino. Ademais, as variáveis independentes, juntas, influenciaram $65 \%$ da variância dos escores da subescala $\operatorname{Dieta}\left(F_{(1,67)}=2944 ; p=0,001\right)$.

Tabela 2. Regressão linear múltipla utilizando a BRUMS, o BSQ e o percentual de gordura como variáveis explicativas da variância da subescala Dieta do EAT-26 em jovens atletas do sexo feminino.

\begin{tabular}{ccccccc}
\hline Variável & Bloco & $B$ & $\mathbf{R}$ & $\mathbf{R}^{\mathbf{2}}$ & $\mathbf{R}^{2 *}$ & $\begin{array}{c}p \\
\text { valor }\end{array}$ \\
\hline BRUMS & 1 & 0,09 & 0,21 & 0,05 & 0,04 & n.s. \\
BSQ & 2 & 0,17 & 0,79 & 0,63 & 0,61 & $\leq 0,01$ \\
$\% \mathrm{G}$ & 3 & 0,86 & 0,55 & 0,30 & 0,27 & $\leq 0,03$ \\
Todos & & 11.44 & 0,80 & 0,65 & 0,55 & $\leq 0,01$ \\
\hline
\end{tabular}

R2* = R2 ajustado; BRUMS = Escala de Humor de Brunel; $B S Q=$ Body Shape Questionnaire; $\% \mathrm{G}=$ Percentual de gordura; n.s. = não significativo para $\mathrm{p}<0,05$.

A Tabela 3 ilustra o modelo de regressão que utilizou a subescala Bulimia e Preocupação com Alimentos como variável critério. Os resultados apontaram a influência estatisticamente significativa do BSQ $\left(F_{(1,67)}=12,38\right.$; $p=0,001)$ e do percentual de gordura $\left(F_{(1,67)}=5,81 ; p=0,04\right)$. Logo, os achados demonstraram que a insatisfação corporal $(48 \%)$ e o percentual de gordura $(30 \%)$ influenciaram a compulsão alimentar e os comportamentos purgativos de atletas de atletismo do sexo feminino. Ressalta-se que a BRUMS $\left(F_{(1,67)}=0,9 ; p=0,74\right)$ não explicou a variância da subescala Bulimia e Preocupação com Alimentos. Todavia, todas as variáveis independentes juntas (BRUMS, BSQ e $\% \mathrm{G})$ impactaram sobre $50 \%\left(F_{(1,67)}=12,70 ; p=0,001\right)$ da variância da subescala Bulimia e Preocupação com Alimentos do EAT-26.

O último modelo de regressão, o qual utilizou a subescala Autocontrole Oral como variável dependente, pode ser

Tabela 3. Regressão linear múltipla utilizando a BRUMS, o BSQ e o percentual de gordura como variáveis explicativas da variância da subescala Bulimia e Preocupação com Alimentos do EAT-26 em jovens atletas do sexo feminino...

\begin{tabular}{ccccccc}
\hline Variável & Bloco & $B$ & $\mathbf{R}$ & $\mathbf{R}^{2}$ & $\mathbf{R}^{2 *}$ & $\begin{array}{c}p \\
\text { valor }\end{array}$ \\
\hline BRUMS & 1 & 0,04 & 0,07 & 0,05 & 0,007 & n.s. \\
BSQ & 2 & 0,12 & 0,69 & 0,48 & 0,45 & $\leq 0,01$ \\
$\% \mathrm{G}$ & 3 & 0,50 & 0,55 & 0,30 & 0,25 & $\leq 0,04$ \\
Todos & & 7,49 & 0,71 & 0,50 & 0,37 & $\leq 0,01$ \\
\hline
\end{tabular}

R2* = R2 ajustado; BRUMS = Escala de Humor de Brunel; BSQ = Body Shape Questionnaire; $\% \mathrm{G}=$ Percentual de gordura; n.s. = não significativo para $\mathrm{p}<0,05$

observado na Tabela 4. Apenas a BRUMS $\left(F_{(1,67)}=4,51\right.$; $p=0,04)$ demonstrou impacto significante $(7 \%)$ sobre os escores da subescala Autocontrole Oral. Nesse sentido, o $\operatorname{BSQ}\left(F_{(1,67)}=2,23 ; p=0,15\right)$ e o percentual de $\operatorname{gordura}\left(F_{(1,67)}=\right.$ $1,26 ; p=0,44)$ não explicaram a variância do autocontrole em relação à comida em jovens atletas de atletismo. Contudo, salienta-se que o modelo de regressão com todas as variáveis explicativas (BRUMS, BSQ e \%G) inseridas em um único

Tabela 4. Regressão linear múltipla utilizando a BRUMS, o BSQ e o percentual de gordura como variáveis explicativas da variância da subescala Autocontrole Oral do EAT-26 em jovens atletas do sexo feminino.

\begin{tabular}{ccccccc}
\hline Variável & Bloco & $B$ & $\mathbf{R}$ & $\mathbf{R}^{\mathbf{2}}$ & $\mathbf{R}^{2 *}$ & $\begin{array}{c}p \\
\text { valor }\end{array}$ \\
\hline BRUMS & 1 & 0,11 & 0,26 & 0,07 & 0,06 & $\leq 0,04$ \\
BSQ & 2 & 0,08 & 0,38 & 0,14 & 0,10 & n.s. \\
$\% \mathrm{G}$ & 3 & 0,32 & 0,23 & 0,05 & 0,03 & n.s. \\
Todos & & 11,92 & 0,26 & 0,08 & 0,06 & n.s. \\
\hline
\end{tabular}

R2* = R2 ajustado; BRUMS = Escala de Humor de Brunel; BSQ = Body Shape Questionnaire; $\% \mathrm{G}=$ Percentual de gordura; n.s. = não significativo para $\mathrm{p}<0,05$.

bloco $\left(F_{(1,67)}=5,01 ; p=0,03\right)$ modulou $8 \%$ da variância da subescala Autocontrole Oral do EAT-26.

No que concerne às comparações das subescalas do EAT-26 em razão dos grupos de humor, alguns achados merecem destaque (Tabela 5): 1) não foi identificada diferença de escore na subescala Dieta entre as atletas com alto e baixo DTH $\left.\left(F_{(2,66)}=1,13 ; p=0,69\right) ; 2\right)$ as atletas com alto e baixo DTH demonstraram semelhanças nos escores da subescala Bulimia e Preocupação com Alimentos $\left(F_{(2,66)}=\right.$ $1,04 ; p=0,73)$; e 3 ) as jovens atletas com alto DTH foram evidenciadas com maiores escores na subescala Autocontrole Oral quando comparadas as esportistas com baixo DTH $\left(\mathrm{Fi}_{2}\right.$, $\left.{ }_{66)}=8,13 ; p=0,03\right)$. 
Tabela 5. Comparação dos escores das subescalas do EAT-26 em função dos grupos de humor em jovens atletas do sexo feminino.

\begin{tabular}{cccc}
\hline Variável & \multicolumn{2}{c}{ Grupo } & $p$ valor \\
\hline & $\begin{array}{c}\text { Baixo DTH } \\
(\text { BRUMS }<101)\end{array}$ & $\begin{array}{c}\text { Alto DTH } \\
(\text { BRUMS } \geq 101)\end{array}$ \\
Mieta & $5,61( \pm 1,08)$ & $4,99( \pm 1,17)$ & n.s. \\
Bul e PA & $2,21( \pm 0,80)$ & $2,55( \pm 0,87)$ & n.s. \\
AC Oral & $2,24( \pm 1,13)$ & $3,59( \pm 1,33)$ & $\leq 0,03$ \\
\hline
\end{tabular}

Bul e $\mathrm{PA}=$ Bulimia e Preocupação com Alimentos; $\mathrm{AC}=$ Autocontrole; BRUMS $=$ Escala de Humor de Brunel; DP = Desvio padrão; EAT-26 = Eating Attitudes Test; n.s. = não significativo para $\mathrm{p}<0,05$.

\section{Discussão}

A presente pesquisa teve como objetivo analisar os efeitos do estado de humor sobre os CAI em jovens atletas do sexo feminino. Os resultados demonstraram prevalência moderada (14\%) para os CAI (EAT+). Esses achados são corroborados por outras investigações conduzidas com atletas brasileiros do sexo feminino (Fortes, Almeida, Laus et al., 2012; Fortes, Oliveira et al., 2012). Parece que a prevalência de CAI em atletas competidoras de esportes de potência (e.g., atletismo) costuma ser relativamente baixa (Fortes \& Ferreira, 2011; Gomes et al., 2011). De fato, a literatura científica não aponta atletas de atletismo como candidatas a TA (De Bruin et al., 2007; Fortes \& Ferreira, 2011).

Por outro lado, a literatura aponta que a insatisfação corporal acomete tanto as atletas quanto as não atletas (Hoch et al., 2009; Reiking \& Alexander, 2005). Rosendahl et al. (2009) explicam que as atletas são expostas às mesmas mensagens socioculturais direcionadas para a magreza que as não atletas. As influências midiáticas e familiares referentes ao peso e a forma corporal, somadas à subcultura esportiva, podem gerar sentimentos depreciativos com o peso e a aparência física nas atletas (Fortes et al., 2013; Fortes, Oliveira et al., 2012). Essa possibilidade encontra apoio em inúmeros estudos que mostram prevalência relativamente elevada (e.g., 25 a 40\%) de insatisfação corporal em atletas do sexo feminino, independente da modalidade esportiva avaliada (De Bruin et al., 2007; Fortes et al., 2013; Fortes $\&$ Ferreira, 2011), o que corrobora os achados da presente investigação.

No que tange o primeiro modelo de regressão (Subescala Dieta), os resultados demonstraram que $63 \%$ e $30 \%$ da variância da restrição alimentar foi explicada pelos escores do BSQ e pelo percentual de gordura, respectivamente. No entanto, não foi evidenciada influência da BRUMS na subescala Dieta do EAT-26. De fato, esses achados apontaram que o DTH não impactou os períodos longos sem ingestão de alimentos com alto teor calórico por atletas do sexo feminino. As meninas atletas costumam restringir a ingestão alimentar com o intuito de reduzir o peso corporal e maximizar o desempenho esportivo (De Bruin et al., 2007). Nesse sentido, parece que o estado de humor não possui relação com a adoção de condutas alimentares restritivas no âmbito esportivo. Logo, uma atleta com elevado DTH não necessariamente estará vulnerável para a restrição alimentar. Evidências demonstram que o DTH pode ser alterado em função da intensidade de treinamento físico ou do desempenho esportivo obtido em competição (Vieira et al., 2010; Werneck \& Navarro, 2011). Todavia, de acordo com os resultados da presente pesquisa, o estado de humor não é determinante para a adoção de comportamento alimentar restritivo.

Concernente à influência da insatisfação corporal sobre a restrição alimentar, outros estudos indicam achados semelhantes. Por exemplo, Rouveix, Bouget, Pannafieux, Champely e Filaire (2007) avaliaram judocas francesas e identificaram que a satisfação com o peso impactou significativamente os escores da subescala Dieta do EAT26. Em adição, Coelho et al. (2010) confeccionaram uma revisão a respeito dos riscos para os TA em atletas do sexo feminino e evidenciaram que a insatisfação corporal é um dos fatores determinantes para os comportamentos alimentares restritivos. Da mesma forma, outros dados da literatura científica são semelhantes aos do presente estudo no que tange à influência do percentual de gordura sobre os comportamentos de restrição alimentar (De Bruin et al., 2007; Fortes, Oliveira et al., 2012). Segundo Fortes e Ferreira (2011), essa tendência pode ser explicada em razão da gordura corporal ser o principal aspecto morfológico depreciado na cultura ocidental.

Em relação ao modelo de regressão utilizando a subescala Bulimia como variável critério, os resultados indicaram influência somente da insatisfação corporal e do percentual de gordura. Ao contrário do que era esperado, o DTH não influenciou os comportamentos alimentares purgativos e compulsivos em atletas do sexo feminino. Em contrapartida, alguns pesquisadores argumentam que alguns estados de humor (e.g., depressão e ansiedade) podem gerar condutas alimentares compulsivas (Gomes et al., 2011). De acordo com Fortes, Almeida, Laus et al. (2012), os fatores negativos do humor podem tornar a atleta mais susceptível para os comportamentos bulímicos. Desse modo, a ingestão compulsiva de alimentos ricos em carboidratos (e.g., doces) pode induzir a secreção de alguns neurotransmissores associados a alterações positivas no humor, por exemplo, a serotonina. Por conseguinte, atletas com sentimentos de desconforto poderão utilizar métodos purgativos (e.g., autoindução de vômitos) com o intuito de não aumentar o seu peso corporal.

Contudo, novamente a insatisfação corporal e o percentual de gordura demonstraram ser importantes características determinantes para os CAI. No modelo de regressão da Tabela 3 , os escores do BSQ explicaram $48 \%$, enquanto o percentual de gordura influenciou $30 \%$ da variância das pontuações da subescala Bulimia e Preocupação com Alimentos. De fato, pesquisadores ressaltam que a insatisfação com o peso e a aparência física é o principal fator de risco para os sintomas de bulimia em atletas do sexo feminino (Durme et al., 2012; Haase, 2011). Acrescentando, a adiposidade corporal elevada é considerada uma das principais variáveis desencadeadoras para os comportamentos alimentares compulsivos no sexo feminino (De Bruin et al., 2007; Schaal et al., 2011).

No que concerne ao último modelo de regressão, os resultados indicaram influência somente do DTH sobre os escores da subescala Autocontrole Oral do EAT-26. Esses achados demonstram que as atletas com alto DTH são 
influenciadas por forças ambientas, por exemplo, pais, amigos e treinadores, para controlarem os tipos e as quantidades de alimentos que costumam ingerir. Fortes, Almeida, Laus et al. (2012) salientam que as jovens que apresentam alterações negativas no humor podem aprender com os treinadores e familiares os conceitos de alimentos "saudáveis" e "menos saudáveis" com maior facilidade quando comparadas às adolescentes com humor positivo.

Por outro lado, a insatisfação corporal e o percentual de gordura não impactaram significativamente os escores da subescala Autocontrole Oral. Entretanto, De Bruin et al. (2007) afirmam que as jovens insatisfeitas são mais facilmente influenciadas por forças ambientais do que as adolescentes satisfeitas com o corpo. Logo, sabendo da relação do percentual de gordura com a insatisfação corporal, era de se esperar que essas variáveis influenciassem a variância da subescala Autocontrole Oral no presente estudo. Nessa linha de raciocínio, Fortes, Oliveira et al. (2012) ressaltam que as atletas com o percentual de gordura elevado estão mais vulneráveis a adotar comportamentos alimentares deletérios a saúde, apontados como ideais pelos treinadores para otimizar o desempenho esportivo. Sendo assim, mais investigações são necessárias para esclarecer melhor esse tópico.

A respeito da comparação dos escores das subescalas do EAT-26 em razão dos grupos de DTH estabelecidos a partir da mediana da BRUMS, a MANCOVA indicou diferenças apenas para a subescala Autocontrole Oral. Esses achados evidenciaram que a frequência de restrição alimentar e de comportamentos alimentares compulsivos e purgativos foram semelhantes entre as atletas com alto e baixo DTH. Em contrapartida, o teste post hoc de Bonferroni apontou diferenças para a subescala Autocontrole Oral. Desse modo, evidenciou-se que as atletas com elevado humor negativo foram mais influenciadas por forças ambientais (e.g., treinadores, pais e amigos) para o controle da ingestão alimentar quando comparadas às atletas com baixo DTH, corroborando os apontamentos de Fortes, Almeida, Laus et al. (2012).

Embora esta investigação demonstre resultados inéditos, faz-se necessário mencionar algumas limitações. Aponta-se o baixo tamanho amostral apresentado, o que impossibilita uma generalização para a população. Destaca-se também a utilização de método duplamente indireto para estimar a gordura corporal das atletas. Entretanto, ressalta-se a dificuldade de acesso a equipamentos sofisticados como o DEXA e a ultrassonografia computadorizada, além do alto dispêndio financeiro na utilização desses aparelhos.

Por fim, os resultados encontrados neste estudo permitem concluir que o DTH influenciou somente o autocontrole oral sobre os tipos e as quantidades de alimentos ingeridos em atletas do sexo feminino. Nesse sentido, evidenciou-se que as esportistas com humor negativo elevado podem estar mais susceptíveis à influência ambiental para a ingestão alimentar. Em suma, jovens atletas, caso demonstrem alterações negativas do humor, podem estar mais vulneráveis para o desencadeamento dos TA.

Outro achado interessante foi o impacto do percentual de gordura e da insatisfação corporal sobre os CAI. Dessa forma, concluiu-se que a morfologia corporal, bem como o sentimento de desgosto com o próprio corpo contribuíram para acentuar a frequência de CAI em jovens atletas competidoras de diversas provas do atletismo.

\section{Referências}

Bighetti, F., Santos, C. B., Santos, J. E., \& Ribeiro, R. P. P. (2004). Translation and validation of the Eating Attitudes Test in female adolescentes in Ribeirão Preto, SP, Brazil. Jornal Brasileiro de Psiquiatria, 53(6), 339-346.

Coelho, G. M., Soares, E. A., \& Ribeiro, B. G. (2010). Are female athletes at increased risk for disordered eating and its complications? Appetite, 55(3), 379-387.

Conti, M. A., Cordás, T. A., \& Latorre, M. R. D. O. (2009). A study of the validity and reliability of the Brazilian version of the Body Shape Questionnaire (BSQ) among adolescents. Revista Brasileira de Saúde Materna e Infantil, 9(3), 331-338.

Costarelli, V., \& Stamou, D. (2009). Emotional intelligence, body image and disordered eating attitudes in combat sport athletes. Journal of Exercise Science \& Fitness, 7(2), 104-111.

De Bruin, A. P., Oudejans, R. R. D., \& Bakker, F. C. (2007). Dieting and body image in aesthetic sports: A comparison of Dutch female gymnasts and non-aesthetic sport participants. Psychology of Sport and Exercise, 8(4), 507-520.

Durme, K. V., Goossens, L., \& Braet, C. (2012). Adolescent aesthetic athletes: A group at risk for eating pathology? Eating Behaviors, 13(1), 119-122.

Filaire, E., Rouveix, M., Pannafieux, C., \& Ferrand, C. (2007). Eating attitudes, perfectionism and body-esteem of elite male judoists and cyclists. Journal of Sports Science and Medicine, 6(1), 50-57.

Fortes, L. S., Almeida, S. S., \& Ferreira, M. E. C. (2012). Maturation process, body dissatisfaction and inappropriate eating behavior in young athletes. Revista de Nutrição, 25(5), 575-586.

Fortes, L. S., Almeida, S. S., Laus, M. F., \& Ferreira, M. E. C. (2012). Level of psychological commitment to exercise and comparison of body dissatisfaction of athletes participating in the Pan-American school games. Revista Brasileira de Medicina do Esporte, 18(4), 238-241.

Fortes, L. S., \& Ferreira, M. E. C. (2011). Comparison of body dissatisfaction and inappropriate eating behavior in adolescent athletes of different sports. Revista Brasileira de Educação Física e Esporte, 25(4), 707-716.

Fortes, L. S., Oliveira, F. G., \& Ferreira, M. E. C. (2012). Influence of affective, anthropometric and sociodemographic factors on eating behavior in young athletes. Jornal Brasileiro de Psiquiatria, 61(3), 148-153.

Fortes, L. S., Neves, C. M., Filgueiras, J. F., Almeida, S. S., \& Ferreira, M. E. C. (2013). Body dissatisfaction, psychological commitment to exercise and eating behavior in young athletes of aesthetic sports. Revista Brasileira de Cineantropometria e Desempenho Humano. 15(16), 695-704.

Gomes, A. R., Martins, C., \& Silva, L. (2011). Eating disordered behaviors in Portuguese athletes: The influence of personal, sport, and psychological variables. European Eating Disorders Review, 19(1), 190-200. 
Haase, A. M. (2011). Weight perception in female athletes: Associations with disordered eating correlates and behavior. Eating Behaviors, 12(1), 64-67.

Hoch, A. Z., Pajewski, N. M., Moraski, L., Carrera, G. F., Wilson, C. R., Hoffmann, R. G., . . Gutterman, D. D. (2009). Prevalence of the female athlete triad in high school athletes and sedentary students. Clinical Journal Sport Medicine, 19(5), 421-428.

The Internacional Society for Advancement for Kineanthropometry (ISAK) (2012). Body Composition in Sport, Exercise and Health. Londres: Routledge.

Reiking, M. F., \& Alexander, L. E. (2005). Prevalence of disorderedeating behaviors in undergraduate female collegiate athletes and nonathletes. Journal of Athletic Training, 40(1), 47-51.

Rohlfs, I. C. P. M., Rotta, T. M., Luft, C. D. B., Andrade, A., Krebs, R. J., \& Carvalho, T. (2008). A Escala de Humor de Brunel (BRUMS): instrumento para detecção precoce da síndrome do excesso de treinamento. Revista Brasileira de Medicina do Esporte, 14(3), 176-181.

Rosendahl, J., Bormann, B., Aschenbrenner, K., Aschenbrenner, F., \& Strauss, B. (2009). Dieting and disordered eating in German high school athletes and non-athletes. Scandinavian Journal of Medicine \& Science in Sports, 19(4), 731-739.

Rouveix, M., Bouget, M., Pannafieux, C., Champely, S., \& Filaire, E. (2007). Eating attitudes, body esteem, perfectionism and anxiety of judo athletes and nonathletes. International Journal of Sports Medicine, 28(2), 340-345.
Schaal, K., Tafflet, M., Nassif, H., Thibault, V., Pichard, C., Alcotte, M., ... Toussaint, J. (2011). Psychological balance in high-level athletes: Gender-based differences and sport-specific patterns. Psychopathology and High Level Sport, 6(5), 1-9.

Scoffier, S., Woodman, T., \& D`Arripe-Longueville, F. (2011). Psychosocial consequences of disordered eating attitudes in elite female figure skaters. European Eating Disorders Review, 19(3), 280-287.

Slaughter, M. H., Lohman, T. G., Boileau, R., Hoswill, C. A., Stillman, R. J., \& Yanloan, M. D. (1988). Skinfold equations for estimation of body fatness in children and youth. Human Biology, 60(3), 709-723.

Vieira, L. F., Oliveira, J. S., Gaion, P. A., Oliveira, H. G., Rocha, P. G. M., \& Vieira, J. L. L. (2010). Mood states and training: A study with high performance deep runners athletes. Revista da Educação Física/UEM, 21(4), 585-591.

Werneck, F. Z., \& Navarro, C. A. (2011). Physical activity level and state of mood in adolescents. Psicologia: Teoria e Pesquisa, 27(2), 189-193.
Recebido em 24.04.2013

Primeira decisão editorial em 15.01.2014

Versão final em 20.01.2014

Aceito em 28.02.2014 